\title{
OTHONOMICS
}

Revista de economía, empresa y sociedad

TRABAJO FINAL MBA. PLAN DE EMPRESA

\section{Lluerna, un modelo de negocio social para la electrificación rural}

\section{Daniel Gaballé}

Executive MBA (UOC)

\section{Alexandre Mollá}

MBA Social Entrepreneurship (UOC)

\section{Gil Blanch}

MBA Social Entrepreneurship (UOC)

RESUMEN Lluerna es un plan de empresa social desarrollado como trabajo final ${ }^{1}$ del ciclo de estudios de MBA por parte de estudiantes de la especialidad de emprendimiento social y de executive MBA de la UOC.

Como resultado de esta colaboración, el plan combina técnicas clásicas de escuela de negocios con enfoques más innovadores propios del emprendimiento social, centrando la actividad de la empresa en el impacto social y no solo en la rentabilidad económica. Así, este plan de negocio constituye un ejemplo de la posibilidad de crear empresas con beneficios pero con un objetivo social, en este caso, la electrificación rural.

En este sentido, el plan muestra un modelo de negocio rentable y aplicable en varios países con bajos índices de electrificación en el ámbito rural, que, además, tiene un fuerte impacto positivo en la vida de las personas. El caso concreto de Lluerna está centrado en Bolivia, donde además de existir las condiciones de mercado adecuadas hay una importante voluntad de colaboración por parte de los distintos actores locales en el mundo de la electrificación rural.

Lluerna pone de manifiesto, pues, cómo la combinación de nuevas tecnologías y de nuevas formas de gestión comercial puede generar impacto incluso con los que normalmente son considerados demasiado pobres como para formar parte del mercado.

PALABRAS CLAVE base de la pirámide; emprendimiento social; microfranquicia; sostenibilidad; cambio

1. Depositado en el Repositorio Institucional de la UOC (O2) http://hdl.handle.net/10609/41083, el portal que recoge, difunde y preserva las publicaciones digitales en acceso abierto de los miembros de la UOC, elaboradas en el desarrollo de sus actividades de investigación, de docencia y de gestión. 


\title{
Lluerna, a social business model for rural electrification
}

\begin{abstract}
Lluerna is a business plan developed by students from both the UOC's Executive $M B A$ course and its MBA in Social Entrepreneurship course.
\end{abstract}

This work combines classical techniques from business schools with a more innovative approach related to impact-centred (rather than profit-centred) businesses. This business plan demonstrates that it is possible to run a sustainable company with a social objective, in this case the electrification of rural areas.

Furthermore, the plan shows a profitable business model that can be exported to many countries, where it is possible to create a market with a remarkable positive impact on a great number of families. Specifically, Lluerna is implemented in Bolivia, a country with an important lack of rural electrification, and where significant levels of collaboration from local actors in the area of rural electrification can be found.

Lluerna is also an example of how putting together new technologies and new commerce management tools makes it possible to generate a positive impact, even with a population that is normally considered too poor to be part of the market.

KEYWORDS base of the pyramid; social entrepreneurship; microfranchise; sustainability; change

\section{Introducción}

En Bolivia hay 1,3 millones de personas sin acceso a la red de distribución de energía eléctrica. La gran mayoría de esta población se concentra en comunidades rurales aisladas, donde el despliegue de la red convencional se encuentra con dificultades importantes debido a su elevado coste y bajo retorno económico.

El perfil de la población sin acceso a la red eléctrica está marcado por un nivel de pobreza más elevado respecto a la media de la población boliviana, junto con un bajo nivel de formación. En las zonas rurales, las limitaciones en el acceso a los servicios básicos incrementan la precariedad de las comunidades.

Lluerna pretende dar respuesta a esta realidad, facilitando el acceso a la energía eléctrica a particulares situados fuera de la cobertura de la red de distribución de energía eléctrica convencional en Bolivia a través del suministro y la instalación de dispositivos de generación de energía de bajo coste basados en energía solar.

Así, Lluerna tiene como misión sustituir el uso de pilas, velas y otras fuentes ineficientes de energía por dispositivos portátiles más eficientes, económicos y duraderos en las comunidades rurales de Bolivia, mejorando la calidad de vida de la población rural, que constituye la visión y el verdadero objetivo final del proyecto.

En este sentido, Lluerna se propone alcanzar un 10\% de la población objetivo (unos 45.000 hogares) en un plazo de 5 años. Este objetivo significaría un aumento del 25\% de la energía consumida por los usuarios, mientras que su inversión en energía bajaría en un 35\% respecto a los valores actuales.

Para conseguir este objetivo, es necesaria la captación de un volumen de financiación de $200.000 €$, que permitirían cubrir las fases iniciales de arranque y crecimiento de la empresa, generando beneficios a partir del quinto año. El potencial de venta del mercado, superior a los 100 millones de euros, permite ofrecer un retorno de inversión muy interesante a medio plazo, lo que facilita la captación de inversores. 


\section{Análisis de necesidades y definición de los objetivos}

\section{Perfil de demanda energética de la población objetivo}

La falta de acceso de la población rural de Bolivia a sistemas convencionales de energía se debe principalmente a la elevada dispersión de estas comunidades, que -junto con la orografía y la poca accesibilidad de las zonas andinas y subandinas- aumenta enormemente los costes de instalación de las infraestructuras necesarias para tener una red de distribución clásica.

En palabras de Miquel Fernández, director ejecutivo de la Plataforma Energética: «Mientras la población urbana de Bolivia vive ya en el siglo XXI, la población rural, dispersa y aislada, aún vive una realidad del siglo XIX. Dos siglos es la diferencia entre el campo y la ciudad».

Las demandas para usos no térmicos representan únicamente el 10\% de la demanda energética de las poblaciones rurales. Electricidad, queroseno, velas, pilas y gasóleo se invierten principalmente en iluminación (5\%) y en el acceso a los medios de comunicación (2\%), dos usos de gran impacto en la calidad de vida rural pero que no requieren grandes cantidades de energía, por lo que se pueden cubrir con equipos portátiles de baja potencia que no necesitan infraestructura y que se pueden conseguir a bajo coste.

Para confirmar este mapa de demanda se ha contactado con organizaciones conocedoras del terreno. De acuerdo con estas organizaciones, el patrón de demanda de la población en zonas rurales sin acceso a la red de distribución eléctrica convencional es muy similar al de la población en zonas periurbanas, que a pesar de tener acceso a la red eléctrica presenta un perfil de ingresos similar. Este perfil es el siguiente:

\begin{tabular}{|l|l|}
\hline Iluminación & $100 \%$ de los hogares \\
\hline Carga de teléfono móvil & $95 \%$ de los hogares \\
\hline Radio & $95 \%$ de los hogares \\
\hline Televisión & $70 \%$ de los hogares \\
\hline Nevera & $2 \%$ de los hogares, siempre vinculado a un uso comercial \\
\hline
\end{tabular}

Lluerna pretende dar respuesta a las tres funcionalidades que se utilizan en el 95\% de los hogares utilizando equipos solares domésticos de última generación, aprovechando los altos índices de radiación solar que registran la región del altiplano y la zona interandina de Bolivia.

\section{El mercado boliviano de sistemas solares domésticos}

El mercado boliviano de sistemas solares domésticos es relativamente antiguo, ya que comenzó a desarrollarse a finales de los años ochenta con las tecnologías disponibles en aquel momento sobre la base de un programa de desarrollo financiado por la Unión Europea, que dotó de tecnología solar en una proporción muy importante a las escuelas rurales de Bolivia.

La aparición de esta tecnología a ojos de muchas familias rurales provocó una publicidad indirecta muy importante (toda la población tenía el deseo de tener la misma tecnología que la escuela), lo que creó un nuevo mercado.

Hasta mediados de los años noventa existió en Bolivia un mercado privado de sistemas solares domésticos (conocidos como SHS por sus siglas en inglés) que daban salida a las funciones básicas de iluminación y radio pero que resultaban caros (alrededor de unos 500 \$) y poco confiables debido a la tecnología utilizada para las cargas y las baterías. 
En 1995 se creó un programa de desarrollo financiado por Shell y el gobierno holandés, que distribuyó 18.000 SHS con una subvención del 50\%; esta iniciativa bienintencionada tuvo consecuencias nefastas para el mercado privado existente, ya que los cuatro principales distribuidores privados existentes en ese momento no pudieron hacer frente a esta competencia de mercado y abandonaron el negocio.

Al terminar el programa, no quedó en Bolivia ninguna estructura dedicada al servicio técnico de unos sistemas solares que por el tipo de baterías que utilizaban necesitaban reparaciones y sustituciones frecuentes; como consecuencia, la confianza de los compradores en este tipo de sistemas se vio muy reducida y las posibilidades de que apareciera de nuevo un mercado privado se han visto muy mermadas.

Lluerna debe plantearse pues un modelo de negocio que recupere esta confianza, un modelo que no se puede centrar únicamente en la distribución, sino que debe dar respuesta al mantenimiento técnico posterior.

\section{Requisitos de distribución y financiación}

En las zonas rurales de Bolivia una parte muy importante de la población está vinculada a la agricultura y recibe sus ingresos anuales concentrados en el momento de la cosecha. En este sentido, la mayor parte de la población objetivo podría llegar a adquirir los equipos solares sin financiación si su coste es inferior a su excedente de ingresos una vez cubiertas las necesidades básicas.

No obstante, la necesidad de generar confianza en estos equipos solares y en la presencia de un soporte técnico hace recomendable plantear un modelo basado en pagos periódicos (mensuales o trimestrales), donde el cliente ve implícita una garantía de servicio técnico.

La distribución de la población objetivo, caracterizada por un alto grado de dispersión, supone pues uno de los grandes retos que superar en el modelo de negocio. En este sentido, el desarrollo de una red de distribuidores locales es imprescindible para la distribución, el cobro de las cuotas y la oferta de servicio técnico pero también como fuente de información de proximidad hacia los consumidores finales.

\section{Escalabilidad del modelo}

América Latina engloba más de 24 millones de personas sin acceso a la red eléctrica. Lluerna pretende diseñar un modelo escalable que se pueda exportar sin grandes modificaciones a otros países de América Latina que presentan problemáticas y oportunidad similares.

\section{Diseño y desarrollo}

Las necesidades detectadas y los objetivos fijados marcan el diseño de la solución, el diseño de la empresa, la cadena de distribución (que debe actuar también de servicio técnico de primer nivel) y el modo de financiación para mantener el vínculo entre los clientes y Lluerna.

\section{Diseño de la solución}

Aprovechando los desarrollos tecnológicos de los últimos años tanto en tecnología LED como en baterías para pequeños equipos electrónicos que se han convertido en universales (como los teléfonos móviles o los MP3), ha aparecido toda una nueva generación de sistemas solares domésticos.

Los nuevos sistemas SHS deben suministrar energía a cargas de mucha menos potencia, lo que permite utilizar baterías distribuidas de iones de litio (Li-lon) o de litio-hierro-fosfato similares a las de los teléfonos móviles, que presentan un bajo grado de degradación. Son equipos SHS portátiles, económicos, escalables y robustos que necesitan muy poco o nulo mantenimiento durante su vida útil y además operan con paneles solares cinco veces más pequeños que en el pasado reciente. 
Para el proyecto Lluerna se ha elegido un equipo pico solar de un fabricante establecido en Bolivia desde principios de los años noventa. Concretamente, el sistema propuesto se compone de:

1) Un panel solar de $10 \mathrm{~W}$, de pequeño formato gracias a las pequeñas cargas utilizadas.

2) Un distribuidor general que conecta el panel solar a las pico lámparas.

3) Tres pico lámparas, que actúan como puntos de iluminación, una de las cuales se puede utilizar como lámpara de sobremesa o como lámpara de mano en sustitución de una linterna.

4) Dos distribuidores USB para aumentar los puntos de conexión disponibles.

5) Dos interruptores remotos para encender/apagar las lámparas cuando están colgadas del techo.

6) Todo el cableado necesario de interconexión.

Respecto a la financiación del producto, se ofertará un pago de cantidades equivalentes a las que actualmente destina la población objetivo a la adquisición de pilas y velas. Esto permite cubrir el coste del producto en solo tres años, aunque la vida útil del producto es superior a siete años. Para cubrir las garantías de pago se propone una estructura que es habitual en los microcréditos a pequeñas comunidades, donde el grupo avala cada individuo.

En resumen, la oferta de producto de Lluerna se puede resumir en tres puntos clave:

1) Nuestros equipos son económicos, ya que suponen la mitad del coste de equipos similares de primera o segunda generación.

2) Nuestros equipos son escalables y versátiles, pudiéndose ampliar y adaptar en función de las necesidades.

3) Nuestros equipos garantizan una larga vida útil, con puntos de distribución sobre el terreno que no solo actúan como gestores del pago, sino que responden ante posibles fallos técnicos.

\section{Forma jurídica y estructura de la empresa}

La estructura de empresa propuesta responde a la necesidad de mantener una estructura muy ligera que minimice los costes estructurales y operativos; en este sentido, hay múltiples formas jurídicas que permiten iniciar la actividad con una inversión mínima y el hecho de actuar como intermediario entre diferentes actores ya existentes reduce sensiblemente los costes operativos.

Respecto a la distribución, Lluerna propone utilizar los canales ya existentes, principalmente pequeños comerciantes rurales que hoy en día ya ofrecen las pilas y velas que utiliza la población que constituye nuestro mercado objetivo, ofreciéndoles un paquete de microfranquicia que les dé de manera estandarizada y empaquetada los conocimientos y los procesos necesarios para actuar como distribuidores autorizados.

El contenido de la microfranquicia estaría constituido por:

- La posibilidad de posicionarse como distribuidor autorizado de la marca Lluerna.

- Material impreso de presentación y promoción del producto y de imagen de marca.

- Formación técnica sobre el producto y servicio posventa básico.

- Formación financiera para construir la propuesta de financiación y hacer el seguimiento de los pagos.

- Formación logística y gestión de los envíos. 


\section{Implementación}

El calendario de implementación del proyecto debe responder durante el primer año a la necesidad de mantener un buen control de las fases iniciales de vida de la empresa, reduciendo así los riesgos asumidos. En este sentido, se propone el desarrollo de una fase piloto durante el primer año de vida de la empresa, de acuerdo con el siguiente calendario:

- De 0 a 3 meses: verificación sobre el terreno directamente en Bolivia de las estimaciones que hemos hecho en el plan de negocio y crear el plan piloto con los partners locales.

- De 3 a 6 meses: negociación con el Gobierno de Bolivia de posibles ayudas al desarrollo del plan piloto, así como de las condiciones de financiación con entidades financieras y desarrollo de la propuesta para microfranquicias con el conocimiento adquirido sobre el terreno.

- De 6 a 8 meses: cierre de acuerdos con microfranquiciados de la zona piloto y ejecución de la formación.

- De 8 a 12 meses: inicio de la actividad en la zona piloto y primera evaluación de resultados.

Una vez desarrollada la fase piloto, y una vez verificado el cumplimiento de objetivos y certificada la viabilidad del proyecto Lluerna, se iniciaría una fase de expansión en primer término en el resto del territorio boliviano.

Para soportar este despliegue en dos fases se ha desarrollado un plan financiero que considera la cobertura de solo un 1\% del mercado potencial en el primer año (menos de 5.000 hogares) para llegar al 2\% del mercado potencial anualmente durante los siguientes tres años.

El modelo propuesto significa que Lluerna necesita financiar de antemano los sistemas solares que distribuye a través de las microfranquicias y que el beneficio de la venta no se acumulará completamente hasta terminados los pagos por parte del cliente final. Para esta financiación inicial y para mantener los precios de venta lo más bajos posible hemos elegido una fórmula que incluye una subvención a fondo perdido el primer año de una fundación privada dedicada a la ayuda al desarrollo de comunidades locales, y para el segundo y tercer año, créditos de tipo clásico. El plan presenta un equilibrio financiero el segundo y tercer año y beneficios a partir del cuarto.

\section{Evaluación}

Al tratarse de un proyecto social, es muy importante poder medir el impacto del proyecto en la sociedad. Para ello, planteamos los siguientes indicadores:

1) Porcentaje de población objetivo cubierta por el proyecto, calculado a partir del número de unidades vendidas y el censo de familias en la zona.

2) Consumo de energía de cada familia cubierta por proyecto, verificando si la introducción de los nuevos aparatos ha incrementado el consumo de energía medio de la familia.

3) Gasto asociado al consumo energético por cada familia cubierta por el proyecto, confirmando la disminución del gasto con la introducción de los nuevos aparatos.

Estos indicadores permiten, asimismo, conocer aspectos de negocio clave, como el grado de penetración en el mercado, el impacto en el consumidor o el posicionamiento del producto respecto a otros productos del mismo segmento o productos sustitutivos.

Para la obtención de los datos se utilizará la red de distribuidores en el territorio, con los que existe una relación directa y continuada fundamentada en la fase de pilotaje. 


\section{Conclusiones}

La combinación de formas de financiación clásicas con una red de distribución local basada en microfranquicias, así como las nuevas tecnologías de solares y de iluminación LED, permiten proponer un modelo de negocio innovador, que cubre un mercado al que no pueden acceder las empresas tradicionales de distribución de electricidad.

Esta estructura alternativa no solo puede ser rentable, sino que puede constituir un factor muy importante de desarrollo de comunidades rurales, que sin estas tecnologías y nuevos modelos de negocio permanecerían todavía en el siglo XIX

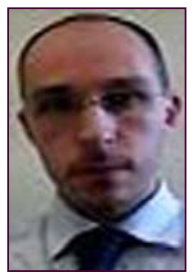

\section{Daniel Gaballé dcaballe@uoc.edu Executive MBA (UOC)}

Licenciado en Física por la Universidad de Barcelona y máster en Administración de empresas por la Universitat Oberta de Catalunya. Su carrera profesional ha estado centrada en la consultoría de dirección estratégica y el desarrollo de sistemas de información, en especial en el ámbito sanitario. Actualmente es gerente en Better Consultants.

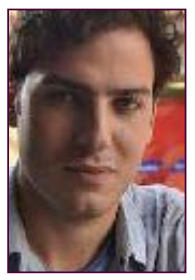

\section{Alexandre Mollá} amollaf@uoc.edu MBA Social Entrepreneurship (UOC)

Licenciado en Psicología y MBA Social Entrepreneurship por la UOC. En el marco de su carrera profesional, cabe destacar su trabajo para Independent Living Alternative como asistente personal de personas independientes pero con alto grado de discapacidad. Actualmente es deputy general manager en Grupo Ensanche.

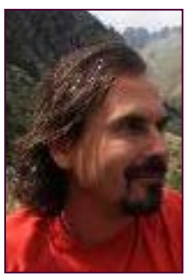

\section{Gil Blanch} gblanch0@uoc.edu MBA Social Entrepreneurship (UOC)

Ingeniero en Automática y Electrónica Industrial y MBA Social Entrepreneurship por la UOC. Su carrera profesional se ha desarrollado tanto en el ámbito de la industria automovilística como, más recientemente, en el sector de generación de energías renovables. Actualmente es gestor de mantenimiento de servicios eólicos en Alstom Power.

Los textos publicados en esta revista están -si no se indica lo contrario- bajo una licencia Reconocimiento-Sin obras derivadas 3.0 España de Creative Commons. Puede copiarlos, distribuirlos y comunicarlos públicamente siempre que cite su autor y la revista y la institución que los publica (autoría, nombre de la revista, institución editora); no haga con ellos obras derivadas. La licencia completa se puede consultar en http://creativecommons.org/licenses/by-nd/3.0/es/deed.es.

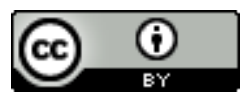

